\title{
A Convenient Approach to a Novel Group of Quaternary Amino Acids Containing a Geminal Bisphosphonate Moiety
}

\author{
Marek Dzięgielewski, Joanna Hejmanowska, Lukasz Albrecht* \\ Institute of Organic Chemistry, Department of Chemistry, Lodz University of Technology, Żeromskiego 116, 90-924 Lodz, Poland \\ E-mail: lukasz.albrecht@p.lodz.pl \\ Received: 29.05.2014; Accepted after revision: 25.07.2014
}

\begin{abstract}
Quaternary amino acids containing a geminal bisphosphonate moiety have been synthesized for the first time. The developed two-step reaction sequence utilizes the Michael addition of $\alpha$ substituted azlactones to a vinylidene bisphosphonate as the key step. The reaction proceeds under catalytic conditions with excellent regioselectivity. Subsequent, acid-mediated azlactone ring opening affords the target quaternary amino acids with good overall yields. Attempts to develop an enantioselective version of the synthetic strategy are described.
\end{abstract}

Key words: azlactones, quaternary amino acids, geminal bisphosphonates, Michael addition, biologically relevant molecules

Target-oriented synthesis of specific structural motives important for medicinal chemistry constitutes an important goal in modern organic chemistry. ${ }^{1}$ Among different biologically relevant molecules, quaternary amino acids occupy a prominent position due to their interesting biological properties (Scheme 1). ${ }^{2}$ For example, incorporation of this structural motif into biomolecules can result in improved biostability due to the presence of the quaternary carbon atom. ${ }^{3}$ Geminal bisphosphonates constitute another important group of pharmacologically active compounds that have found application in the treatment of several bone disorders such as Paget's disease, myeloma, bone metastases, or osteoporosis. ${ }^{4}$ As both groups of compounds have interesting biological properties, it was anticipated that merging their pharmacological potential may lead to the development of a novel class of biologically active molecules.

$\alpha$-Substituted azlactones constitute an important group of quaternary amino acid precursors that have found widespread application in contemporary organic synthesis. ${ }^{5}$ Their ability to undergo Michael additions is well recognized. ${ }^{6}$ Surprisingly, to the best of our knowledge no reports on Michael addition of azlactones to phosphoryl group stabilized Michael acceptors exists in the literature. We designed a route that incorporated a quaternary amino acid moiety into the side chain of a geminal bisphosphonate which relied on the Michael addition of an $\alpha$-substituted azlactone to a vinylidene bisphosphonate? However, at the outset of our studies important challenges related to the regioselectivity of the reaction had to be considered. It is well recognized that azlactones display

SYNTHESIS 2014, 46, 3233-3238

Advanced online publication: 26.08 .2014

DOI: 10.1055/s-0034-1378997; Art ID: ss-2014-z0330-op

C Georg Thieme Verlag Stuttgart · New York nucleophilicity both in the $\mathrm{C} 2$ and $\mathrm{C} 4$ positions (Scheme 2). Furthermore, O-alkylation constitutes another possibility.

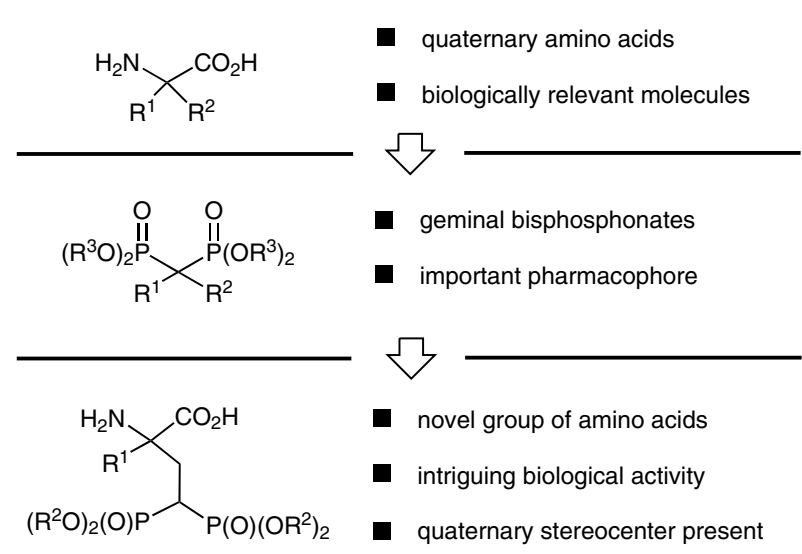

Scheme 1 Relevance of amino acid and geminal bisphosphonate moieties in chemistry and biology

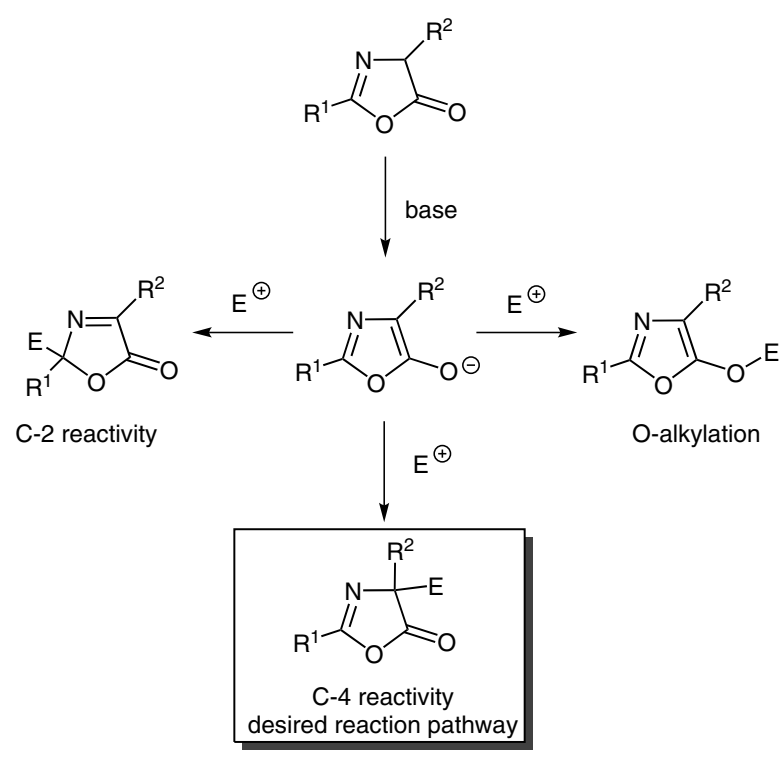

Scheme 2 Reactivity of azlactones

Herein, we report the first example of a Michael reaction between an $\alpha$-substituted azlactone and a vinylidene bisphosphonate (Scheme 3). The developed catalytic approach benefits from operational simplicity and easily available starting materials. The possibility to open the azlactone moiety for the synthesis of quaternary amino acids 
is demonstrated. Furthermore, attempts for the development of an enantioselective version of the reaction employing chiral Brønsted base catalysis were undertaken. Importantly, this reaction results in the formation of quaternary stereocenter containing molecules, which is an important and challenging task in modern organic synthesis. ${ }^{8}$

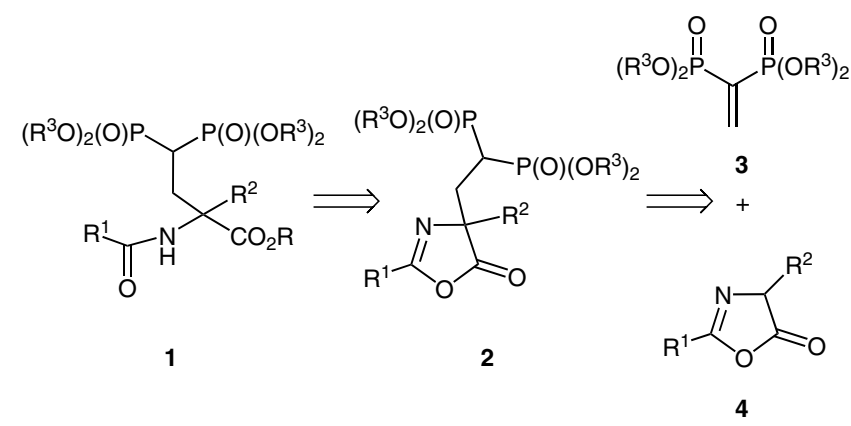

Scheme 3 Novel approach to the quaternary amino acids containing geminal bisphosphonate moiety

Studies were initiated with the goal of finding optimal conditions for the devised Michael addition. Tetraethyl ethene-1,1-diylbis(phosphonate) (3a) was chosen as a model Michael acceptor. Initial screening revealed that the devised Michael reaction can be performed employing a catalytic amount of base. Triethylamine was identified as the best base and dichloromethane as the optimal solvent. Under these conditions the Michael reaction employing $\alpha$-benzyl-substituted azlactone $\mathbf{4 a}$ as a donor was terminated within 24 hours affording $\mathbf{2 a}$ in a high $88 \%$ yield. Under these conditions the scope of the methodology was investigated (Table 1). It was found that various substituents could be present in the $\alpha$-position of starting azlactones $\mathbf{4 a}-\mathbf{f}$. Branching in the alkyl chain of $\mathbf{4 b}-\mathbf{c}$ led to yield deterioration as demonstrated in the synthesis of 2b and 2c. The effect was particularly pronounced in the case of 2c. Simple methyl-substituted azlactone 4d also underwent the Michael reaction, albeit the formation of small amounts of the C2 regioisomer was observed. Good results were obtained with the phenyl-substituted azlactone $\mathbf{4} \mathbf{e}$ demonstrating the possibility of introducing an aromatic moiety at the $\mathrm{C} 2$ position of the target amino acid 2e. Finally, the introduction of a heteroatom in the side chain of the starting azlactone $4 \mathbf{f}$ was attempted. In this case the reaction proceeded efficiently affording $\mathbf{2} \mathbf{f}$ as a single regioisomer in good yield.

Further studies were focused on the transformation of $\alpha, \alpha$ disubstituted azlactones $\mathbf{2 a}-\mathbf{f}$ thus obtained into the desired quaternary amino acids $\mathbf{1 a}-\mathbf{f}$. Gratifyingly, it was found that under standard reaction conditions ${ }^{9}$ the azlactone moiety can be efficiently opened, affording linear amino acids 1a-f bearing the geminal bisphosphonate moiety (Table 2). In all cases moderate to good yields were obtained confirming the generality of the developed synthetic methodology.

The possibility to perform the reaction under basic conditions prompted us to investigate an enantioselective ver-
Table 1 Michael Addition of $\alpha$-Substituted Azlactones 4 to Tetraethyl Ethene-1,1-diylbis(phosphonate) (3a) ${ }^{\mathrm{a}}$

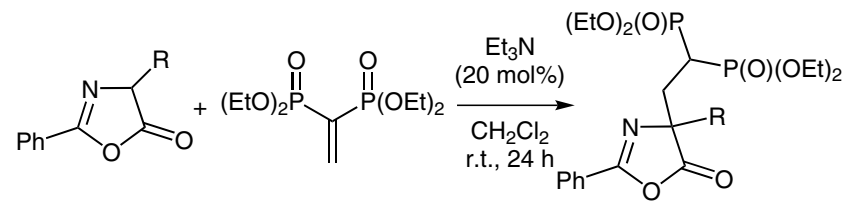

4

$3 a$

\begin{tabular}{llll}
\hline Entry & $\mathrm{R}$ & Regioisomeric ratio $^{\mathrm{b}}$ & Yield (\%) \\
\hline 1 & $\mathrm{Bn}(\mathbf{4 a )}$ & $>20: 1$ & $88(\mathbf{2 a})$ \\
2 & $i$-Bu (4b) & $>20: 1$ & $55(\mathbf{2 b})$ \\
3 & $i$-Pr (4c) & $>20: 1$ & $44(\mathbf{2 c})$ \\
4 & $\mathrm{Me} \mathrm{(4d)}$ & $5: 1$ & $49(\mathbf{2 d})$ \\
5 & $\mathrm{Ph}(\mathbf{4 e})$ & $>20: 1$ & $63(\mathbf{2 e})$ \\
6 & $\mathrm{CH}_{2} \mathrm{CH}_{2} \mathrm{SMe} \mathrm{(4f)}$ & $>20: 1$ & $65(\mathbf{2 f})$ \\
\hline
\end{tabular}

${ }^{a}$ Reactions performed on 0.25 -mmol scale (for details see the experimental section).

${ }^{b}$ Regioisomeric ratio as determined by ${ }^{1} \mathrm{H}$ NMR of a crude reaction mixture.

sion of the reaction employing different chiral Brønsted bases (Scheme 4). Initial studies showed that the use of simple cinchona alkaloid $\mathbf{5 a}$ afforded target adduct $\mathbf{2 b}$ with $37 \%$ ee. Attempts were made to improve the enantioselectivity of the reaction by a simple modification of the catalyst employed. Therefore, different cinchona alkaloids $\mathbf{5 a}, \mathbf{b}$ and their derivatives $\mathbf{5} \mathbf{c}-\mathbf{e}$ were evaluated in the reaction. Disappointingly, cinchonidine $(\mathbf{5 b})$ afforded the target product $\mathbf{2 b}$ as a racemic mixture. Similarly, employment of the bifunctional catalysts $\mathbf{5 c}$ and $\mathbf{5 d}$ bearing double H-bonding moieties led to the deterioration of en-

Table 2 Synthesis of Quaternary Amino Acids via Acid-Mediated Azlactone Ring Opening ${ }^{\mathrm{a}}$

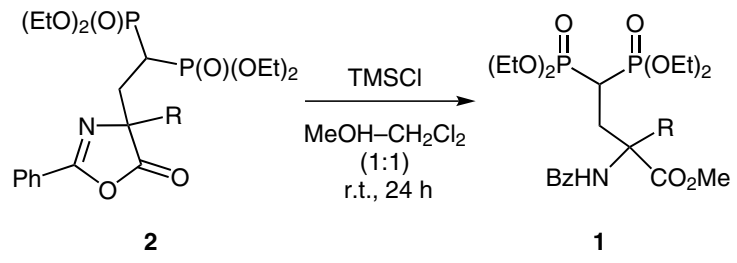

\begin{tabular}{lll}
\hline Entry & $\mathrm{R}$ & Yield (\%) \\
\hline 1 & $\mathrm{Bn}(\mathbf{2 a})$ & $58(\mathbf{1 a})$ \\
2 & $i-\mathrm{Bu}(2 \mathbf{b})$ & $53(\mathbf{1 b})$ \\
3 & $i-\operatorname{Pr}(\mathbf{2 c})$ & $51(\mathbf{1 c})$ \\
4 & $\mathrm{Me} \mathrm{(2d)}$ & $47(\mathbf{1 d})$ \\
5 & $\mathrm{Ph}(\mathbf{2 e})$ & $43(\mathbf{1})$ \\
6 & $\mathrm{CH}_{2} \mathrm{CH}_{2} \mathrm{SMe}(\mathbf{2 f})$ & $56(\mathbf{1 f})$ \\
\hline
\end{tabular}

a Reactions performed on 0.1-mmol scale (for details see the experimental section). 


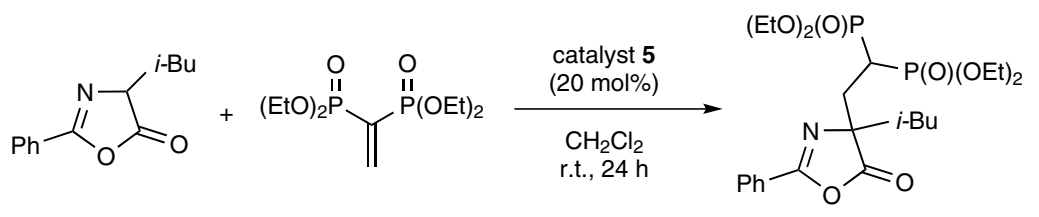

$4 b$

$3 \mathbf{a}$

ent-2b

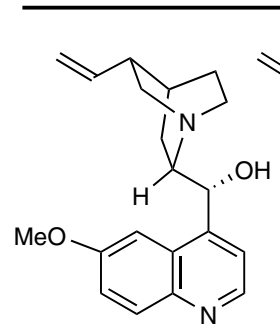

$\mathbf{5 a}$
$60 \%, 37 \%$ ee<smiles>C=CC1CC2CCCN(C2)C1[C@H](O)c1ccnc2ccccc12</smiles>

$5 \mathbf{b}$ $49 \%, 3 \%$ ee<smiles>C=CC1CN2CCC1C2(C)C(NC(=S)NN)c1ccnc2ccc(OC)cc12</smiles>

$5 \mathrm{c}$

$\mathrm{Ar}=3,5-\left(\mathrm{CF}_{3}\right)_{2} \mathrm{C}_{6} \mathrm{H}_{3}$ $55 \%, 30 \%$ ee

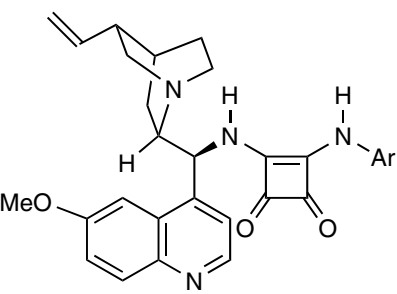

$5 d$

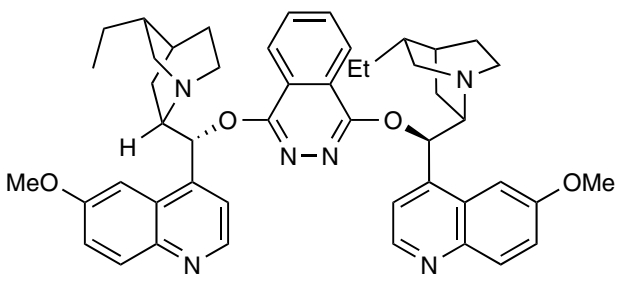

5 e

$50 \%, 15 \%$ ee

Scheme 4 Asymmetric synthesis of quaternary amino acid $\mathbf{2 b}$

antioselectivity. Furthermore, the use of dimeric cinchona alkaloid catalyst 5e did not lead to an improvement in the enantioselectivity of the reaction. Further studies on the enantioselective version of the reaction are ongoing in our laboratories.

In conclusion, we have developed a new approach to a new class of $\alpha, \alpha$-disubstituted $\alpha$-amino acid. It utilizes a two-step reaction sequence consisting of Michael addition of $\alpha$-substituted azlactones 4 to tetraethyl ethene-1,1-diylbis(phosphonate) (3a) followed by azlactone ring opening under acidic conditions. The first step of the developed synthetic strategy could be performed under basic, catalytic conditions. Initial attempts to develop an enantioselective version of this reaction have been demonstrated. The presence of a geminal bisphosphonate moiety in the side chain of the target amino acids may lead to products with interesting biological properties.

Azlactones 4a-f and tetraethyl ethene-1,1-diylbis(phosphonate) (3a) were prepared according to literature procedures. ${ }^{10}{ }^{1} \mathrm{H}$ and ${ }^{13} \mathrm{C}$ NMR spectra were recorded on a Bruker Ultra Shield 700 instrument. ${ }^{1} \mathrm{H}$ NMR with the solvent reference relative to TMS employed as the internal standard $\left(\mathrm{CDCl}_{3}, \delta=7.26\right) ;{ }^{13} \mathrm{C}$ NMR relative to TMS with the respective solvent resonance as the internal standard $\left(\mathrm{CDCl}_{3}, \delta=77.16\right) .{ }^{31} \mathrm{P}$ NMR spectra were recorded either on a Bruker Ultra Shield 700 or Bruker Avance DPX 250 spectrometer at r.t. in $\mathrm{CDCl}_{3}$ as a solvent using $85 \% \mathrm{H}_{3} \mathrm{PO}_{4}$ as external standard. Flash chromatography was performed on Merck silica gel (60, par- ticle size: $35-70 \mu \mathrm{m}$ ). TLC was carried out on commercially available pre-coated plates (Silica on TLC Alu foils, Fluka) and visualized by UV light. Catalysts, which are commercially available reagents, were purchased from Aldrich and used as received. Cinchona-derived catalysts $\mathbf{5 c}-\mathbf{d}$ were synthesized following the procedures reported in the literature. ${ }^{11}$ Enantiomeric excess of adduct $\mathbf{2 b}$ was determined by chiral stationary phase HPLC using Daicel Chiralpak IC column.

\section{Michael Addition of $\alpha$-Substituted Azlactones 4 to Tetraethyl} Ethene-1,1-diylbis(phosphonate) (3a); General Procedure An ordinary screw-cap vial was charged with a magnetic stirring bar, $\mathrm{CH}_{2} \mathrm{Cl}_{2}(1 \mathrm{~mL})$, tetraethyl vinylbisphosphonate (3a, $0.25 \mathrm{mmol}$, 1 equiv) and $\mathrm{Et}_{3} \mathrm{~N}$ (0.05 mmol, 0.2 equiv). Subsequently, azlactone $4(0.25 \mathrm{mmol}, 1$ equiv) was added and the resulting mixture was stirred at r.t. for $24 \mathrm{~h}$. After this time the mixture was directly subjected to flash column chromatography (silica gel) to afford target product 2 .

Tetraethyl [2-(4-Benzyl-5-oxo-2-phenyl-4,5-dihydrooxazol-4yl)ethane-1,1-diyl]bis(phosphonate) (2a)

Following the general procedure using (EtOAc- $\mathrm{MeOH}, 95: 5$ ) gave 2a as a single regioisomer as a pale yellow oil; yield: $121.8 \mathrm{mg}$ $(88 \%)$.

${ }^{1} \mathrm{H}$ NMR $\left(700 \mathrm{MHz}, \mathrm{CDCl}_{3}\right): \delta=7.83-7.81(\mathrm{~m}, 2 \mathrm{H}), 7.53-7.49(\mathrm{~m}$, $1 \mathrm{H}), 7.41(\mathrm{t}, J=7.8 \mathrm{~Hz}, 2 \mathrm{H}), 7.14-7.11(\mathrm{~m}, 5 \mathrm{H}), 4.22-4.14(\mathrm{~m}, 4$ H), 4.13-4.05 (m, $4 \mathrm{H}), 3.18$ (d, $J=13.4 \mathrm{~Hz}, 1 \mathrm{H}), 3.15$ (d, $J=13.4$ $\mathrm{Hz}, 1 \mathrm{H}), 2.83-2.62(\mathrm{~m}, 3 \mathrm{H}), 1.35(\mathrm{t}, J=7.0 \mathrm{~Hz}, 3 \mathrm{H}), 1.34(\mathrm{t}, J=$ $6.9 \mathrm{~Hz}, 3 \mathrm{H}), 1.24(\mathrm{t}, J=7.1 \mathrm{~Hz}, 6 \mathrm{H})$.

${ }^{13} \mathrm{C} \mathrm{NMR}\left(176 \mathrm{MHz}, \mathrm{CDCl}_{3}\right): \delta=178.8,160.8,133.7,132.5,130.4$ (2 C), 128.6 (2 C), 128.0 (2 C), 127.8 (2 C), 127.3, 125.8, 72.3 (dd, $J=9.3,2.4 \mathrm{~Hz}), 63.0(\mathrm{~d}, J=6.4 \mathrm{~Hz}), 62.8(\mathrm{~d}, J=7.4 \mathrm{~Hz}), 62.7(\mathrm{~d}$, 
$J=7.7 \mathrm{~Hz}), 62.7(\mathrm{~d}, J=7.1 \mathrm{~Hz}), 45.4,32.3(\mathrm{dd}, J=135.3,132.7$ $\mathrm{Hz}), 32.0$ (t, $J=3.9 \mathrm{~Hz}), 16.4(\mathrm{~d}, J=6.5 \mathrm{~Hz}), 16.3(\mathrm{~d}, J=6.4 \mathrm{~Hz})$, $16.2(\mathrm{~d}, J=5.5 \mathrm{~Hz}, 2 \mathrm{C})$.

${ }^{31} \mathrm{P}$ NMR $\left(101 \mathrm{MHz}, \mathrm{CDCl}_{3}\right): \delta=23.96,23.63$.

HRMS: $m / z[\mathrm{M}+\mathrm{H}]^{+}$calcd for $\mathrm{C}_{26} \mathrm{H}_{36} \mathrm{NO}_{8} \mathrm{P}_{2}$ : 552.1911; found: 552.1915 .

Tetraethyl [2-(4-Isobutyl-5-oxo-2-phenyl-4,5-dihydrooxazol-4yl)ethane-1,1-diyl]bis(phosphonate) (2b)

Following the general procedure (EtOAc-MeOH, 95:5) gave $\mathbf{2 b}$ as a single regioisomer as a pale yellow oil; yield: $71.3 \mathrm{mg}(55 \%)$.

${ }^{1} \mathrm{H}$ NMR $\left(700 \mathrm{MHz}, \mathrm{CDCl}_{3}\right): \delta=8.01-7.99(\mathrm{~m}, 2 \mathrm{H}), 7.58-7.55(\mathrm{~m}$, $1 \mathrm{H}), 7.48(\mathrm{t}, J=7.8 \mathrm{~Hz}, 2 \mathrm{H}), 4.20-4.13(\mathrm{~m}, 4 \mathrm{H}), 4.13-4.04(\mathrm{~m}, 4$ $\mathrm{H}), 2.70$ (dddd, $J=25.3,23.8,6.6,3.2 \mathrm{~Hz}, 1 \mathrm{H}), 2.61-2.45(\mathrm{~m}, 2 \mathrm{H})$, $1.91(\mathrm{dd}, J=14.2,5.7 \mathrm{~Hz}, 1 \mathrm{H}), 1.81(\mathrm{dd}, J=14.1,6.4 \mathrm{~Hz}, 1 \mathrm{H})$, 1.58 (nonet, $J=13.0 \mathrm{~Hz}, 1 \mathrm{H}), 1.33(\mathrm{q}, J=6.9 \mathrm{~Hz}, 6 \mathrm{H}), 1.24(\mathrm{dt}$, $J=8.3,7.1 \mathrm{~Hz}, 6 \mathrm{H}), 0.87(\mathrm{~d}, J=6.6 \mathrm{~Hz}, 6 \mathrm{H})$.

${ }^{13} \mathrm{C} \mathrm{NMR}\left(176 \mathrm{MHz}, \mathrm{CDCl}_{3}\right): \delta=180.1,160.4,132.7,128.8(2 \mathrm{C})$, $127.9(2 \mathrm{C}), 126.0,70.8(\mathrm{dd}, J=7.9,3.0 \mathrm{~Hz}), 62.9(\mathrm{~d}, J=6.5 \mathrm{~Hz})$, $62.7(\mathrm{~d}, J=6.5 \mathrm{~Hz}, 3 \mathrm{C}), 47.6,33.0(\mathrm{t}, J=4.3 \mathrm{~Hz}), 31.9(\mathrm{~d}, J=135.4$, $134.7 \mathrm{~Hz}), 24.7,24.1,23.6,16.3(\mathrm{~d}, J=6.2 \mathrm{~Hz}), 16.3(\mathrm{~d}, J=6.2 \mathrm{~Hz})$, $16.2(\mathrm{~d}, J=5.7 \mathrm{~Hz}), 16.2(\mathrm{~d}, J=5.7 \mathrm{~Hz})$.

${ }^{31} \mathrm{P}$ NMR $\left(101 \mathrm{MHz}, \mathrm{CDCl}_{3}\right): \delta=24.08,23.78$.

HRMS: $m / z[\mathrm{M}+\mathrm{H}]^{+}$calcd for $\mathrm{C}_{23} \mathrm{H}_{38} \mathrm{NO}_{8} \mathrm{P}_{2}$ : 518.2067; found: 518.2072 .

Tetraethyl [2-(4-Isopropyl-5-oxo-2-phenyl-4,5-dihydrooxazol4-yl)ethane-1,1-diyl]bis(phosphonate) (2c)

Following the general procedure (EtOAc- $\mathrm{MeOH}, 95: 5)$ gave $\mathbf{2 c}$ as a single regioisomer as a pale yellow oil; yield: $55.0 \mathrm{mg}(44 \%)$.

${ }^{1} \mathrm{H}$ NMR (700 MHz, $\left.\mathrm{CDCl}_{3}\right): \delta=8.01-7.99(\mathrm{~m}, 2 \mathrm{H}), 7.58-7.54(\mathrm{~m}$, $1 \mathrm{H}), 7.49-7.45$ (m, $2 \mathrm{H}), 4.21-4.14(\mathrm{~m}, 4 \mathrm{H}), 4.13-4.01(\mathrm{~m}, 4 \mathrm{H})$, $2.73-2.55(\mathrm{~m}, 3 \mathrm{H}), 2.08$ (hept, $J=6.8 \mathrm{~Hz}, 1 \mathrm{H}), 1.34(\mathrm{t}, J=7.1, \mathrm{~Hz}$, $3 \mathrm{H}), 1.34(\mathrm{t}, J=7.3 \mathrm{~Hz}, 3 \mathrm{H}), 1.25(\mathrm{t}, J=7.1 \mathrm{~Hz}, 3 \mathrm{H}), 1.22(\mathrm{t}, J=$ $7.1 \mathrm{~Hz}, 3 \mathrm{H}), 1.02(\mathrm{~d}, J=6.8 \mathrm{~Hz}, 3 \mathrm{H}), 0.98(\mathrm{~d}, J=6.8 \mathrm{~Hz}, 3 \mathrm{H})$.

${ }^{13} \mathrm{C} \mathrm{NMR}\left(176 \mathrm{MHz}, \mathrm{CDCl}_{3}\right): \delta=179.3,161.0,132.6,128.7(2 \mathrm{C})$, 127.9 (2 C), 126.0, 73.3 (d, $J=9.6 \mathrm{~Hz}), 62.9(\mathrm{~d}, J=6.2 \mathrm{~Hz}), 62.7$ $(\mathrm{d}, J=5.9 \mathrm{~Hz}, 2 \mathrm{C}), 62.6(\mathrm{~d}, J=5.1 \mathrm{~Hz}), 37.2,32.1(\mathrm{dd}, J=135.7$, $133.5 \mathrm{~Hz}), 29.9$ (t, $J=3.9 \mathrm{~Hz}), 16.7,16.4,16.4(\mathrm{~d}, J=6.4 \mathrm{~Hz}), 16.3$ $(\mathrm{d}, J=6.5 \mathrm{~Hz}), 16.2(\mathrm{~d}, J=5.9 \mathrm{~Hz}), 16.2(\mathrm{~d}, J=5.6 \mathrm{~Hz})$.

${ }^{31} \mathrm{P}$ NMR (101 MHz, $\left.\mathrm{CDCl}_{3}\right): \delta=24.25,24.03$.

HRMS: $m / z[\mathrm{M}+\mathrm{H}]^{+}$calcd for $\mathrm{C}_{22} \mathrm{H}_{36} \mathrm{NO}_{8} \mathrm{P}_{2}: 504.1911$; found: 504.1919 .

Tetraethyl [2-(4-Methyl-5-oxo-2-phenyl-4,5-dihydrooxazol-4yl)ethane-1,1-diyl]bis(phosphonate) (2d)

Following the general procedure (EtOAc-MeOH, 95:5) gave 2d as a mixture of regioisomers as a yellow oil; yield: $58.5 \mathrm{mg}(49 \%)$; ratio 5:1.

${ }^{1} \mathrm{H}$ NMR (700 MHz, $\left.\mathrm{CDCl}_{3}\right): \delta=8.01-7.97(\mathrm{~m}, 2 \mathrm{H}), 7.58-7.55(\mathrm{~m}$, $1 \mathrm{H}), 7.49-7.46$ (m, $2 \mathrm{H}), 4.22-4.15$ (m, $4 \mathrm{H}), 4.14-4.05$ (m, $4 \mathrm{H})$, 2.72 (dddd, $J=25.7,23.5,7.4,2.9 \mathrm{~Hz}, 1 \mathrm{H}), 2.61$ (ddt, $J=18.6$, 15.1, 7.4 Hz, $1 \mathrm{H}$ ), 2.50 (dddd, $J=23.0,19.7,15.2,3.0 \mathrm{~Hz}, 1 \mathrm{H}$ ), $1.53(\mathrm{~s}, 3 \mathrm{H}), 1.35(\mathrm{t}, J=6.7 \mathrm{~Hz}, 3 \mathrm{H}), 1.34(\mathrm{t}, J=6.9 \mathrm{~Hz}, 3 \mathrm{H}), 1.25$ $(\mathrm{t}, J=7.2 \mathrm{~Hz}, 3 \mathrm{H}), 1.24(\mathrm{t}, J=7.2 \mathrm{~Hz}, 3 \mathrm{H})$.

${ }^{13} \mathrm{C} \mathrm{NMR}\left(176 \mathrm{MHz}, \mathrm{CDCl}_{3}\right): \delta=180.0,160.9,132.9,128.9(2 \mathrm{C})$, $128.1(2 \mathrm{C}), 126.2,67.3(\mathrm{dd}, J=9.2,2.9 \mathrm{~Hz}), 63.1(\mathrm{~d}, J=6.7 \mathrm{~Hz})$, $62.9(\mathrm{~d}, J=6.7 \mathrm{~Hz}), 62.8(\mathrm{~d}, J=6.4 \mathrm{~Hz}, 2 \mathrm{C}), 32.9(\mathrm{t}, J=4.3 \mathrm{~Hz})$, $32.3(\mathrm{dd}, J=135.1,133.3 \mathrm{~Hz}), 26.3,16.5(\mathrm{~d}, J=6.4 \mathrm{~Hz}), 16.5$ (d, $J=6.3 \mathrm{~Hz}), 16.4(\mathrm{~d}, J=5.3 \mathrm{~Hz}), 16.4(\mathrm{~d}, J=5.1 \mathrm{~Hz})$.

${ }^{31} \mathrm{P} \mathrm{NMR}\left(283 \mathrm{MHz}, \mathrm{CDCl}_{3}\right): \delta=23.12,22.86$.

HRMS: $m / z[\mathrm{M}+\mathrm{H}]^{+}$calcd for $\mathrm{C}_{20} \mathrm{H}_{32} \mathrm{NO}_{8} \mathrm{P}_{2}$ : 476.1632; found: 476.1639 .
Tetraethyl [2-(5-Oxo-2,4-diphenyl-4,5-dihydrooxazol-4-yl)ethane-1,1-diyl]bis(phosphonate) (2e)

Following the general procedure (EtOAc-MeOH, 95:5) gave 2e as a single regioisomer as a pale yellow oil; yield: $84.4 \mathrm{mg}(63 \%)$.

${ }^{1} \mathrm{H}$ NMR $\left(700 \mathrm{MHz}, \mathrm{CDCl}_{3}\right): \delta=8.10-8.07(\mathrm{~m}, 2 \mathrm{H}), 7.68-7.64(\mathrm{~m}$, $2 \mathrm{H}), 7.61-7.57$ (m, $1 \mathrm{H}), 7.52-7.48(\mathrm{~m}, 2 \mathrm{H}), 7.37-7.33(\mathrm{~m}, 2 \mathrm{H})$, 7.31-7.28 (m, 1 H), 4.15-4.05 (m, 8 H), 3.04-2.94 (m, $1 \mathrm{H}), 2.92-$ $2.73(\mathrm{~m}, 2 \mathrm{H}), 1.29(\mathrm{t}, J=7.1 \mathrm{~Hz}, 3 \mathrm{H}), 1.27(\mathrm{t}, J=7.1, \mathrm{~Hz}, 3 \mathrm{H})$, $1.26(\mathrm{t}, J=7.1, \mathrm{~Hz}, 3 \mathrm{H}), 1.25(\mathrm{t}, J=6.9, \mathrm{~Hz}, 3 \mathrm{H})$.

${ }^{13} \mathrm{C} \mathrm{NMR}\left(176 \mathrm{MHz}, \mathrm{CDCl}_{3}\right): \delta=177.8,161.3,139.7,132.9,128.8$ (2 C), 128.7 (2 C), 128.3, 128.1 (2 C), 125.9, 125.5 (2 C), 71.7 (d, $J=9.5 \mathrm{~Hz}), 62.8(\mathrm{~d}, J=6.2 \mathrm{~Hz}), 62.7(\mathrm{~d}, J=7.3 \mathrm{~Hz}), 62.7(\mathrm{~d}, J=$ $6.5 \mathrm{~Hz}, 2 \mathrm{C}), 35.6(\mathrm{t}, J=4.1 \mathrm{~Hz}), 32.6(\mathrm{t}, J=133.8 \mathrm{~Hz}), 16.3(\mathrm{~d}, J=$ $6.2 \mathrm{~Hz}), 16.3(\mathrm{~d}, J=5.7 \mathrm{~Hz}, 2 \mathrm{C}), 16.2(\mathrm{~d}, J=5.6 \mathrm{~Hz})$.

${ }^{31} \mathrm{P}$ NMR (101 MHz, $\left.\mathrm{CDCl}_{3}\right): \delta=23.58$.

HRMS: $m / z[\mathrm{M}+\mathrm{H}]^{+}$calcd for $\mathrm{C}_{25} \mathrm{H}_{34} \mathrm{NO}_{8} \mathrm{P}_{2}$ : 538.1755; found: 538.1758 .

\section{Tetraethyl (2-\{4-[2-(Methylthio)ethyl]-5-oxo-2-phenyl-4,5-di-} hydrooxazol-4-yl ethane-1,1-diyl)bis(phosphonate) (2f) Following the general procedure (EtOAc-MeOH, 95:5) gave $\mathbf{2 f}$ as a single regioisomer as a colorless oil; yield: $87.3 \mathrm{mg}(65 \%)$.

${ }^{1} \mathrm{H}$ NMR $\left(700 \mathrm{MHz}, \mathrm{CDCl}_{3}\right): \delta=8.02-7.98(\mathrm{~m}, 2 \mathrm{H}), 7.60-7.56(\mathrm{~m}$, $1 \mathrm{H}), 7.50-7.46(\mathrm{~m}, 2 \mathrm{H}), 4.21-4.14(\mathrm{~m}, 4 \mathrm{H}), 4.13-4.03(\mathrm{~m}, 4 \mathrm{H})$, 2.72 (dddd, $J=25.3,23.7,7.1,3.0 \mathrm{~Hz}, 1 \mathrm{H}), 2.64-2.47(\mathrm{~m}, 2 \mathrm{H})$, 2.42 (ddd, $J=12.9,9.7,5.8 \mathrm{~Hz}, 1 \mathrm{H}), 2.34$ (ddd, $J=12.9,10.3,6.4$ $\mathrm{Hz}, 1 \mathrm{H}), 2.25-2.15(\mathrm{~m}, 2 \mathrm{H}), 2.04(\mathrm{~s}, 3 \mathrm{H}), 1.34(\mathrm{t}, J=6.9 \mathrm{~Hz}, 3 \mathrm{H})$, $1.33(\mathrm{t}, J=6.9 \mathrm{~Hz}, 3 \mathrm{H}), 1.25(\mathrm{t}, J=7.1 \mathrm{~Hz}, 3 \mathrm{H}), 1.23(\mathrm{t}, J=7.0$ $\mathrm{Hz}, 3 \mathrm{H})$.

${ }^{13} \mathrm{C} \mathrm{NMR}\left(176 \mathrm{MHz}, \mathrm{CDCl}_{3}\right): \delta=179.3,161.5,133.0,128.9(2 \mathrm{C})$, $128.2(2 \mathrm{C}), 125.9,70.4$ (dd, $J=8.7,2.8 \mathrm{~Hz}), 63.1(\mathrm{~d}, J=6.3 \mathrm{~Hz})$, $62.9(\mathrm{~d}, J=6.2 \mathrm{~Hz}, 2 \mathrm{C}), 62.9(\mathrm{~d}, J=4.6 \mathrm{~Hz}), 38.5,32.4(\mathrm{t}, J=3.8$ $\mathrm{Hz}), 32.0$ (dd, $J=135.7,133.3 \mathrm{~Hz}), 28.2,16.5$ (d, $J=6.5 \mathrm{~Hz}), 16.5$ $(\mathrm{d}, J=6.3 \mathrm{~Hz}), 16.4(\mathrm{~d}, J=5.2 \mathrm{~Hz}), 16.3(\mathrm{~d}, J=5.2 \mathrm{~Hz}), 15.5$.

${ }^{31} \mathrm{P} \mathrm{NMR}\left(283 \mathrm{MHz}, \mathrm{CDCl}_{3}\right): \delta=23.03,22.81$.

HRMS: $m / z[\mathrm{M}+\mathrm{H}]^{+}$calcd for $\mathrm{C}_{22} \mathrm{H}_{36} \mathrm{NO}_{8} \mathrm{P}_{2} \mathrm{~S}$ : 536.1631; found: 536.1637 .

Synthesis of Quaternary Amino Acids via Acid-Mediated Azlactone Ring Opening; General Procedure

An ordinary screw-cap vial was charged with a magnetic stirring bar, the corresponding azlactone $2\left(0.1 \mathrm{mmol}, 1\right.$ equiv), and $\mathrm{CH}_{2-}$ $\mathrm{Cl}_{2}-\mathrm{MeOH}(1: 1,0.4 \mathrm{~mL})$. Subsequently, TMSCl (0.2 mmol, 2 equiv) was added at $0{ }^{\circ} \mathrm{C}$ and the mixture was allowed to warm to r.t. Reaction progress was monitored by TLC. After $24 \mathrm{~h}$ the solvent was evaporated under reduced pressure and the residue was directly subjected to flash chromatography (silica gel) to afford target product 1 .

Methyl 2-Benzamido-2-benzyl-4,4-bis(diethoxyphosphoryl)butanoate (1a)

Following the general procedure (EtOAc-hexane 9:1) gave 1a as a pale yellow oil; yield: $33.6 \mathrm{mg}(58 \%)$.

${ }^{1} \mathrm{H} \mathrm{NMR}\left(700 \mathrm{MHz}, \mathrm{CDCl}_{3}\right): \delta=8.68(\mathrm{~s}, 1 \mathrm{H}), 7.98-7.93(\mathrm{~m}, 2 \mathrm{H})$, 7.51-7.45 (m, 1 H), 7.44-7.41 (m, 2 H), 7.21-7.16 (m, 3 H), 7.09$7.05(\mathrm{~m}, 2 \mathrm{H}), 4.19-4.03(\mathrm{~m}, 6 \mathrm{H}), 4.00-3.90(\mathrm{~m}, 3 \mathrm{H}), 3.81(\mathrm{~s}, 3 \mathrm{H})$, $3.17(\mathrm{~d}, J=14.1 \mathrm{~Hz}, 1 \mathrm{H}), 3.10-3.00(\mathrm{~m}, 1 \mathrm{H}), 2.41-2.31(\mathrm{~m}, 1 \mathrm{H})$, 2.25 (dddd, $J=21.3,18.3,15.8,2.3 \mathrm{~Hz}, 1 \mathrm{H}), 1.34(\mathrm{t}, J=7.2 \mathrm{~Hz}, 3$ $\mathrm{H}), 1.32(\mathrm{t}, J=7.0 \mathrm{~Hz}, 3 \mathrm{H}), 1.25(\mathrm{t}, J=7.1 \mathrm{~Hz}, 3 \mathrm{H}), 1.17(\mathrm{t}, J=$ 7.1 Hz, $3 \mathrm{H})$.

${ }^{13} \mathrm{C} \mathrm{NMR}\left(176 \mathrm{MHz}, \mathrm{CDCl}_{3}\right): \delta=172.5,167.2,136.4,133.9,131.6$, 131.1 (2 C), 128.5 (2 C), 128.1 (2 C), 127.7 (2 C), 126.9, 63.8 (d, $J=6.8 \mathrm{~Hz}), 63.4(\mathrm{~d}, J=6.5 \mathrm{~Hz}), 63.0(\mathrm{~d}, J=6.7 \mathrm{~Hz}), 62.9(\mathrm{~d}, J=$ $6.8 \mathrm{~Hz}), 61.3(\mathrm{dd}, J=6.8,1.6 \mathrm{~Hz}), 52.7,39.4,30.5(\mathrm{t}, J=134.6 \mathrm{~Hz})$, $29.9(\mathrm{t}, J=3.5 \mathrm{~Hz}), 16.6(\mathrm{~d}, J=5.7 \mathrm{~Hz}), 16.5(\mathrm{~d}, J=6.0 \mathrm{~Hz}), 16.4$ $(\mathrm{d}, J=5.7 \mathrm{~Hz}), 16.3(\mathrm{~d}, J=5.4 \mathrm{~Hz})$. 
${ }^{31} \mathrm{P}$ NMR (283 MHz, $\left.\mathrm{CDCl}_{3}\right): \delta=26.27(\mathrm{~d}, J=6.5 \mathrm{~Hz}), 22.96(\mathrm{~d}$, $J=6.5 \mathrm{~Hz}$ ).

HRMS: $m / z[\mathrm{M}+\mathrm{H}]^{+}$calcd for $\mathrm{C}_{27} \mathrm{H}_{40} \mathrm{NO}_{9} \mathrm{P}_{2}$ : 584.2173; found: 584.2179 .

Methyl 2-Benzamido-2-[2,2-bis(diethoxyphosphoryl)ethyl]-4methylpentanoate (1b)

Following the general procedure (EtOAc-hexane 9:1) gave $\mathbf{1 b}$ as a yellow oil; yield: $29.1 \mathrm{mg}(53 \%)$.

${ }^{1} \mathrm{H}$ NMR $\left(700 \mathrm{MHz}, \mathrm{CDCl}_{3}\right): \delta=8.91(\mathrm{~s}, 1 \mathrm{H}), 8.01-7.99(\mathrm{~m}, 2 \mathrm{H})$, $7.49-7.45(\mathrm{~m}, 1 \mathrm{H}), 7.44-7.40(\mathrm{~m}, 2 \mathrm{H}), 4.31-4.22(\mathrm{~m}, 2 \mathrm{H}), 4.18$ $4.06(\mathrm{~m}, 6 \mathrm{H}), 3.75$ (s, $3 \mathrm{H}), 3.05$ (tdd, $J=23.6,6.3,2.5 \mathrm{~Hz}, 1 \mathrm{H})$, 2.72 (dddd, $J=21.8,15.8,8.0,6.3 \mathrm{~Hz}, 1 \mathrm{H}), 2.46$ (dd, $J=14.8,6.9$ $\mathrm{Hz}, 1 \mathrm{H}), 2.23$ (dddd, $J=21.5,18.7,15.7,2.6 \mathrm{~Hz}, 1 \mathrm{H}), 1.91$ (dd, $J=14.8,5.2 \mathrm{~Hz}, 1 \mathrm{H}), 1.71$ (hd, $J=6.8,5.2 \mathrm{~Hz}, 1 \mathrm{H}), 1.38(\mathrm{t}, J=$ $7.1 \mathrm{~Hz}, 3 \mathrm{H}), 1.33(\mathrm{t}, J=7.1 \mathrm{~Hz}, 3 \mathrm{H}), 1.32(\mathrm{t}, J=7.1 \mathrm{~Hz}, 6 \mathrm{H}), 0.98$ $(\mathrm{d}, J=6.7 \mathrm{~Hz}, 3 \mathrm{H}), 0.86(\mathrm{~d}, J=6.6 \mathrm{~Hz}, 3 \mathrm{H})$.

${ }^{13} \mathrm{C}$ NMR $\left(176 \mathrm{MHz}, \mathrm{CDCl}_{3}\right): \delta=173.1,166.7,134.0,131.5,128.5$ $(2 \mathrm{C}), 127.6(2 \mathrm{C}), 63.7(\mathrm{~d}, J=7.0 \mathrm{~Hz}), 63.5(\mathrm{~d}, J=6.4 \mathrm{~Hz}), 63.0(\mathrm{~d}$, $J=6.6 \mathrm{~Hz}), 62.9(\mathrm{~d}, J=6.8 \mathrm{~Hz}), 61.6(\mathrm{~d}, J=6.9,2.2 \mathrm{~Hz}), 52.6,42.2$, $31.0(\mathrm{t}, J=133.3 \mathrm{~Hz}), 30.9(\mathrm{t}, J=3.6 \mathrm{~Hz}), 25.0,24.4,23.9,16.6(\mathrm{~d}$, $J=4.4 \mathrm{~Hz}), 16.5(\mathrm{~d}, J=4.4 \mathrm{~Hz}), 16.5(\mathrm{~d}, J=6.2 \mathrm{~Hz}), 16.4(\mathrm{~d}, J=$ $5.9 \mathrm{~Hz})$.

${ }^{31} \mathrm{P}$ NMR $\left(283 \mathrm{MHz}, \mathrm{CDCl}_{3}\right): \delta=26.82(\mathrm{~d}, J=6.2 \mathrm{~Hz}), 23.10(\mathrm{~d}$, $J=6.2 \mathrm{~Hz})$.

HRMS: $m / z[\mathrm{M}+\mathrm{H}]^{+}$calcd for $\mathrm{C}_{24} \mathrm{H}_{42} \mathrm{NO}_{9} \mathrm{P}_{2}$ : 550.2329; found: 550.2335 .

Methyl 2-Benzamido-4,4-bis(diethoxyphosphoryl)-2-isopropylbutanoate (1c)

Following the general procedure (EtOAc-hexane 9:1) gave 1c as a pale yellow oil; yield: $27.5 \mathrm{mg}(51 \%)$.

${ }^{1} \mathrm{H}$ NMR $\left(700 \mathrm{MHz}, \mathrm{CDCl}_{3}\right): \delta=8.63(\mathrm{~s}, 1 \mathrm{H}), 8.01-7.98(\mathrm{~m}, 2 \mathrm{H})$, 7.49-7.46 (m, 1 H), 7.43-7.40 (m, $2 \mathrm{H}), 4.31-4.22$ (m, $2 \mathrm{H}), 4.18$ $4.04(\mathrm{~m}, 6 \mathrm{H}), 3.75(\mathrm{~s}, 3 \mathrm{H}), 3.08$ (dddd, $J=25.8,23.6,5.9,3.9 \mathrm{~Hz}$, $1 \mathrm{H}), 2.98$ (hept, $J=7.0 \mathrm{~Hz}, 1 \mathrm{H}), 2.54$ (dddd, $J=20.2,15.7,12.0$, $5.9 \mathrm{~Hz}, 1 \mathrm{H}), 2.43$ (dddd, $J=19.7,17.1,15.5,4.0 \mathrm{~Hz}, 1 \mathrm{H}), 1.39(\mathrm{t}$ $J=7.1 \mathrm{~Hz}, 3 \mathrm{H}), 1.34-1.29(\mathrm{~m}, 9 \mathrm{H}), 1.13(\mathrm{~d}, J=6.8 \mathrm{~Hz}, 3 \mathrm{H}), 0.96$ (d, $J=6.9 \mathrm{~Hz}, 3 \mathrm{H})$.

${ }^{13} \mathrm{C} \mathrm{NMR}\left(176 \mathrm{MHz}, \mathrm{CDCl}_{3}\right): \delta=172.3,166.6,134.0,131.5,128.5$ $(2 \mathrm{C}), 127.5(2 \mathrm{C}), 63.8(\mathrm{dd}, J=6.4,4.6 \mathrm{~Hz}), 63.6(\mathrm{~d}, J=7.0 \mathrm{~Hz})$, $63.3(\mathrm{~d}, J=6.6 \mathrm{~Hz}), 62.9(\mathrm{~d}, J=6.7 \mathrm{~Hz}), 62.8(\mathrm{~d}, J=6.9 \mathrm{~Hz}), 52.3$, $31.2(\mathrm{dd}, J=134.0,131.7 \mathrm{~Hz}), 29.8,26.8(\mathrm{t}, J=3.8 \mathrm{~Hz}), 18.6,18.3$, $16.6(\mathrm{~d}, J=5.9 \mathrm{~Hz}, 2 \mathrm{C}), 16.5(\mathrm{~d}, J=6.2 \mathrm{~Hz}), 16.4(\mathrm{~d}, J=5.7 \mathrm{~Hz})$. ${ }^{31} \mathrm{P}$ NMR $\left(283 \mathrm{MHz}, \mathrm{CDCl}_{3}\right): \delta=26.60(\mathrm{~d}, J=5.8 \mathrm{~Hz}), 23.28(\mathrm{~d}$, $J=5.8 \mathrm{~Hz})$.

HRMS: $m / z[\mathrm{M}+\mathrm{H}]^{+}$calcd for $\mathrm{C}_{23} \mathrm{H}_{40} \mathrm{NO}_{9} \mathrm{P}_{2}$ : 536.2173; found: 536.2174 .

\section{Methyl 2-Benzamido-4,4-bis(diethoxyphosphoryl)-2-methylbu- tanoate (1d) \\ Following the general procedure (EtOAc-hexane 9:1) gave 1d as a colorless oil; yield: $23.8 \mathrm{mg}(47 \%)$.}

${ }^{1} \mathrm{H}$ NMR $\left(700 \mathrm{MHz}, \mathrm{CDCl}_{3}\right): \delta=9.14(\mathrm{~s}, 1 \mathrm{H}), 8.07-8.01(\mathrm{~m}, 2 \mathrm{H})$, 7.49-7.43 (m, $1 \mathrm{H}), 7.43-740$ (m, $2 \mathrm{H}$ ), 4.27 (ddq, $J=11.2$, 7.0, 3.8 Hz, $2 \mathrm{H}), 4.21-4.09$ (m, $6 \mathrm{H}), 3.75(\mathrm{~s}, 3 \mathrm{H}), 3.06$ (dddd, $J=26.6$, 24.0, 5.7, 2.8 Hz, $1 \mathrm{H}), 2.47-2.31(\mathrm{~m}, 2 \mathrm{H}), 1.71(\mathrm{~s}, 3 \mathrm{H}), 1.39(\mathrm{t}, J=$ $7.0 \mathrm{~Hz}, 3 \mathrm{H}), 1.34$ (t, $J=7.3 \mathrm{~Hz}, 3 \mathrm{H}), 1.34$ (t, $J=7.1 \mathrm{~Hz}, 3 \mathrm{H}), 1.33$ (t, $J=7.3 \mathrm{~Hz}, 3 \mathrm{H})$.

${ }^{13} \mathrm{C} \mathrm{NMR}\left(176 \mathrm{MHz}, \mathrm{CDCl}_{3}\right): \delta=172.7,166.8,133.6,131.4,128.3$ (2 C), $127.5(2 \mathrm{C}), 63.5(\mathrm{~d}, J=6.5 \mathrm{~Hz}), 63.4(\mathrm{~d}, J=6.0 \mathrm{~Hz}), 62.9(\mathrm{~d}$, $J=7.1 \mathrm{~Hz}), 62.9(\mathrm{~d}, J=7.0 \mathrm{~Hz}), 58.6(\mathrm{dd}, J=6.6,2.1 \mathrm{~Hz}), 52.5$, $33.3(\mathrm{t}, J=3.3 \mathrm{~Hz}), 30.8(\mathrm{dd}, J=133.1,133.0 \mathrm{~Hz}), 23.7,16.4(\mathrm{~d}, J=$
$4.8 \mathrm{~Hz}), 16.4(\mathrm{~d}, J=5.0 \mathrm{~Hz}), 16.3(\mathrm{~d}, J=6.2 \mathrm{~Hz}), 16.3(\mathrm{~d}, J=5.9$ $\mathrm{Hz})$.

${ }^{31} \mathrm{P}$ NMR $\left(283 \mathrm{MHz}, \mathrm{CDCl}_{3}\right): \delta=26.35(\mathrm{~d}, J=6.6 \mathrm{~Hz}), 23.34(\mathrm{~d}$, $J=6.6 \mathrm{~Hz})$.

HRMS: $m / z[\mathrm{M}+\mathrm{H}]^{+}$calcd for $\mathrm{C}_{21} \mathrm{H}_{36} \mathrm{NO}_{9} \mathrm{P}_{2}: 508.1860$; found: 508.1866 .

\section{Methyl 2-Benzamido-4,4-bis(diethoxyphosphoryl)-2-phenylbu-} tanoate (1e)

Following the general procedure (EtOAc-hexane 9:1) gave 1e as a pale yellow oil; yield: $24.4 \mathrm{mg}(43 \%)$.

${ }^{1} \mathrm{H}$ NMR $\left(700 \mathrm{MHz}, \mathrm{CDCl}_{3}\right): \delta=9.59(\mathrm{~s}, 1 \mathrm{H}), 8.16-8.09(\mathrm{~m}, 2 \mathrm{H})$, 7.55-7.52 (m, $2 \mathrm{H}), 7.52-7.50$ (m, $1 \mathrm{H}), 7.49-7.45$ (m, $2 \mathrm{H}), 7.35-$ 7.32 (m, 2 H), 7.28-7.26 (m, 1 H), 4.19-4.07 (m, 7 H), 4.06-3.99 $(\mathrm{m}, 1 \mathrm{H}), 3.75(\mathrm{~s}, 3 \mathrm{H}), 2.96-2.79(\mathrm{~m}, 2 \mathrm{H}), 2.70(\mathrm{tdd}, J=24.6,6.2$, $3.6 \mathrm{~Hz}, 1 \mathrm{H}), 1.33-1.28(\mathrm{~m}, 12 \mathrm{H})$.

${ }^{13} \mathrm{C} \mathrm{NMR}\left(176 \mathrm{MHz}, \mathrm{CDCl}_{3}\right): \delta=171.8,167.0,138.3,133.6,131.8$, 128.5 (2 C), 128.2 (2 C), 127.9 (2 C), 127.7, 127.4 (2 C), 64.8 (dd, $J=7.4,3.6 \mathrm{~Hz}), 63.3(\mathrm{~d}, J=5.1 \mathrm{~Hz}), 63.3(\mathrm{~d}, J=5.7 \mathrm{~Hz}), 63.2(\mathrm{~d}$, $J=6.9 \mathrm{~Hz}), 63.1(\mathrm{~d}, J=6.5 \mathrm{~Hz}), 53.0,34.3(\mathrm{t}, J=3.0 \mathrm{~Hz}), 32.4(\mathrm{dd}$, $J=133.8,131.7 \mathrm{~Hz}), 16.5(\mathrm{~d}, J=5.8 \mathrm{~Hz}), 16.5(\mathrm{~d}, J=4.2 \mathrm{~Hz}), 16.4$ $(\mathrm{d}, J=4.8 \mathrm{~Hz}), 16.4(\mathrm{~d}, J=3.9 \mathrm{~Hz})$.

${ }^{31} \mathrm{P}$ NMR $\left(283 \mathrm{MHz}, \mathrm{CDCl}_{3}\right): \delta=24.28(\mathrm{~d}, J=6.8 \mathrm{~Hz}), 24.04(\mathrm{~d}$, $J=6.8 \mathrm{~Hz})$.

HRMS: $m / z[\mathrm{M}+\mathrm{H}]^{+}$calcd for $\mathrm{C}_{26} \mathrm{H}_{38} \mathrm{NO}_{9} \mathrm{P}_{2}:$ 570.2016; found: 570.2020 .

Methyl 2-Benzamido-4,4-bis(diethoxyphosphoryl)-2-[2-(methylthio)ethyl]butanoate (1f)

Following the general procedure (EtOAc-hexane 9:1) gave 1f as a yellow oil; yield: $31.9 \mathrm{mg}$ (56\%).

${ }^{1} \mathrm{H}$ NMR (700 MHz, $\left.\mathrm{CDCl}_{3}\right): \delta=9.01(\mathrm{~s}, 1 \mathrm{H}), 8.05-7.99(\mathrm{~m}, 2 \mathrm{H})$, $7.51-7.44(\mathrm{~m}, 1 \mathrm{H}), 7.42(\mathrm{dd}, J=8.4,7.0 \mathrm{~Hz}, 2 \mathrm{H}), 4.31-4.23(\mathrm{~m}, 2$ H), 4.18-4.06 (m, $6 \mathrm{H}), 3.77$ (s, $3 \mathrm{H}), 3.06$ (dddd, $J=26.2,23.5,6.4$, $2.2 \mathrm{~Hz}, 1 \mathrm{H}$ ), 2.86 (ddd, $J=14.3,11.4,5.1 \mathrm{~Hz}, 1 \mathrm{H}), 2.65$ (ddt, $J=$ $22.1,15.8,6.7 \mathrm{~Hz}, 1 \mathrm{H}), 2.52$ (ddd, $J=12.9,11.5,5.2 \mathrm{~Hz}, 1 \mathrm{H}), 2.44$ (ddd, $J=12.8,11.0,5.1 \mathrm{~Hz}, 1 \mathrm{H}), 2.31-2.23(\mathrm{~m}, 1 \mathrm{H}), 2.23-2.17(\mathrm{~m}$, $1 \mathrm{H}), 2.06(\mathrm{~s}, 3 \mathrm{H}), 1.39$ (t, $J=7.1 \mathrm{~Hz}, 3 \mathrm{H}), 1.33(\mathrm{t}, J=7.0 \mathrm{~Hz}, 3$ $\mathrm{H}), 1.32(\mathrm{t}, J=7.1 \mathrm{~Hz}, 6 \mathrm{H})$.

${ }^{13} \mathrm{C}$ NMR $\left(176 \mathrm{MHz}, \mathrm{CDCl}_{3}\right): \delta=172.2,166.8,133.6,131.7,128.5$ (2 C), $127.6(2 \mathrm{C}), 63.8(\mathrm{~d}, J=7.3 \mathrm{~Hz}), 63.6(\mathrm{~d}, J=6.5 \mathrm{~Hz}), 63.1$ (d, $J=6.5 \mathrm{~Hz}), 63.0(\mathrm{~d}, J=7.0 \mathrm{~Hz}), 61.2(\mathrm{dd}, J=6.8,1.5 \mathrm{~Hz}), 52.7$, $35.0,30.9(\mathrm{t}, J=133.7 \mathrm{~Hz}), 30.8(\mathrm{t}, J=2.8 \mathrm{~Hz}), 28.8,16.6(\mathrm{~d}, J=$ $3.3 \mathrm{~Hz}), 16.5(\mathrm{~d}, J=3.6 \mathrm{~Hz}), 16.5(\mathrm{~d}, J=6.1 \mathrm{~Hz}), 16.4(\mathrm{~d}, J=5.8$ $\mathrm{Hz}), 15.7$.

${ }^{31} \mathrm{P}$ NMR $\left(283 \mathrm{MHz}, \mathrm{CDCl}_{3}\right): \delta=26.69(\mathrm{~d}, J=7.5 \mathrm{~Hz}), 22.83(\mathrm{~d}$, $J=6.9 \mathrm{~Hz})$.

HRMS: $m / z[\mathrm{M}+\mathrm{H}]^{+}$calcd for $\mathrm{C}_{23} \mathrm{H}_{40} \mathrm{NO}_{9} \mathrm{P}_{2} \mathrm{~S}$ : 568.1894; found: 568.1899 .

Tetraethyl ent-[2-(4-Isobutyl-5-oxo-2-phenyl-4,5-dihydrooxazol-4-yl)ethane-1,1-diyl]bis(phosphonate) (ent-2b) by Enantioselective Michael Addition

An ordinary screw-cap vial was charged with a magnetic stirring bar, $\mathrm{CH}_{2} \mathrm{Cl}_{2}(0.4 \mathrm{~mL})$, tetraethyl vinylbisphosphonate (3a, 0.1 mmol, 1 equiv), and the corresponding catalyst 5 ( $0.02 \mathrm{mmol}, 0.2$ equiv). Subsequently, azlactone $\mathbf{4 b}(0.1 \mathrm{mmol}, 1$ equiv) was added and the resulting mixture was stirred at r.t. for $24 \mathrm{~h}$. After this time the mixture was directly subjected to flash column chromatography (silica gel) to afford target product ent-2b. Spectral data were in accordance with that of racemic $\mathbf{2} \mathbf{b}$. The ee was determined by HPLC (Chiralpak IC column, hexane- $i$-PrOH, 70:30, flow rate 1.0 $\mathrm{mL} / \mathrm{min}$ ): $t_{\mathrm{R}}=26.8$ (major), 14.4 min (minor); $[\alpha]_{\mathrm{D}}{ }^{20}-3.4$ (c 1.14, $\left.\mathrm{CHCl}_{3}\right)$. 


\section{Acknowledgment}

The research was realized within Lider programme from the National Center for Research and Development (NCBR, grant number LIDER/01/87/L3/11/NCBR/2012). ŁA acknowledges Foundation for Polish Science for a supporting grant. The authors are grateful to Dr Jacek Kędzia for providing starting tetraethyl vinylbisphosphonate.

Supporting Information for this article is available online at http://www.thieme-connect.com/products/ejournals/journal/ $10.1055 / \mathrm{s}-00000084$

\section{References}

(1) Schreiber, S. L. Science (Washington, D.C.) 2000, 287, 1964.

(2) (a) Vogt, H.; Bräse, S. Org. Biomol. Chem. 2007, 5, 406. (b) Doyle, A. G.; Jacobsen, E. N. Chem. Rev. 2007, 107, 5713. See also: (c) Cativiela, C.; Diaz-de-Villegas, M. D. Tetrahedron: Asymmetry 2007, 18, 569. (d) Ohfune, Y.; Shinada, T. Eur. J. Org. Chem. 2005, 5127. (e) Najera, C. Synlett 2002, 1388. (f) Cativiela, C.; Diaz-de-Villegas, M. D. Tetrahedron: Asymmetry 2000, 11, 645. (g) Cativiela, C.; Diaz-de-Villegas, M. D. Tetrahedron: Asymmetry 1998, 9 , 3517.

(3) (a) Toniolo, C.; Crisma, M.; Formaggio, F.; Peggion, C. Biopolymers 2001, 60, 396. (b) Kaul, R.; Balaram, P. Bioorg. Med. Chem. 1999, 7, 105. See also: (c) Gilead, S.; Gazit, E. Angew. Chem. Int. Ed. 2004, 43, 4041. (d) Bellier, B.; McCort-Tranchepain, I. I.; Ducos, B.; Danascimento, S.; Meudal, H.; Noble, F.; Garbay, C.; Roques, B. P. J. Med. Chem. 1997, 40, 3947. (e) Burgess, K.; Ho, K.-K.; Pal, B. J. Am. Chem. Soc. 1995, 117, 3808. (f) O'Connor, S. J.; Liu, Z. Synlett 2003, 2135.

(4) (a) Fleish, H. Bisphosphonates in Bone Disease: From the Laboratory to the Patient; Parthenon Publishing Group:
London, 1995. (b) Zhang, S.; Gangal, G.; Uludag, H. Chem. Soc. Rev. 2007, 36, 507. (c) Russell, R. G. G. Bone 2011, 49, 2.

(5) For a review, see: Alba, A-N. R.; Rios, R. Chem. Asian J. 2011, 6, 720.

(6) For selected examples, see: (a) Cabrera, S.; Reyes, E.; Aleman, J.; Milelli, A.; Kobbelgaard, S.; Jørgensen, K. A. J. Am. Chem. Soc. 2008, 130, 12031. (b) Hayashi, Y.; Obi, K.; Ohta, Y.; Okamura, D.; Ishikawa, H. Chem. Asian J. 2009, 4, 246. (c) Alemán, J.; Milelli, A.; Cabrera, S.; Reyes, E.; Jørgensen, K. A. Chem. Eur. J. 2008, 14, 10958.

(d) Balaguer, A. N.; Companyó, X.; Calvet, T.; Font-Bardia, M.; Moyano, A.; Rios, R. Eur. J. Org. Chem. 2009, 199. (e) Alba, A.-N. R.; Companyó, X.; Valero, G.; Moyano, A.; Rios, R. Chem. Eur. J. 2010, 16, 5354. (f) Bravo, N.; Alba, A-N. R.; Valero, G.; Companyó, X.; Moyano, A.; Rios, R. New J. Chem. 2010, 34, 1816.

(7) For application of vinylidene bisphosphonates in organocatalytic Michael additions, see: (a) Sulzer-Mossé, S.; Tissot, M.; Alexakis, A. Org. Lett. 2007, 9, 3749. (b) Sulzer-Mossé, S.; Alexakis, A.; Mareda, J.; Bollot, G.; Bernardinelli, G.; Filinchuk, Y. Chem. Eur. J. 2009, 15, 3204. (c) Barros, M. T.; Phillips, A. M. F. Eur. J. Org. Chem. 2008, 2525. (d) Capuzzi, M.; Perdicchia, D.; Jørgensen, K. A. Chem. Eur. J. 2008, 14, 128. (e) Phillips, A. M. F.; Barros, M. T. Org. Biomol. Chem. 2012, 10, 404. (f) Zhao, M.-X.; Dai, T.-L.; Liu, R.; Wei, D.-K.; Zhou, H.; Ji, F.-H.; Shi, M. Org. Biomol. Chem. 2012, 10, 7970.

(8) Bella, M.; Gasperi, T. Synthesis 2009, 1583.

(9) Jiang, H.; Gschwend, B.; Albrecht, Ł.; Hansen, S. G.; Jørgensen, K. A. Chem. Eur. J. 2011, 17, 9032.

(10) (a) Liang, J.; Ruble, J. C.; Fu, G. C. J. Org. Chem. 1998, 63, 3154. (b) Degenhardt, C. R.; Burdsall, D. C. J. Org. Chem. 1986, 51, 3488.

(11) (a) Vakulya, B.; Varga, S.; Csampai, A.; Soós, T. Org. Lett. 2005, 7, 1967. (b) Yang, W.; Du, D.-M. Org. Lett. 2010, 12, 5450 . 